\title{
SAP Learning Journeys - a double bridge
}

Sven Leukert*

SAP SE, User Assistance CSI, 69190 Walldorf, Germany

\begin{abstract}
SAP Learning Journeys are a key element for providing a guiding path and framework for SAP users and implementation professionals to learn and stay current in their SAP product expertise. They also form a double bridge between the worlds of learning and technical documentation. On one hand, the Learning Journeys themselves are stored and maintained in the DITA content management system used for technical documentation, and then rendered both in the SAP Learning Hub and SAP Help Portal. On the other, the Learning Journeys allow the inclusion and referencing of technical documentation content into the learning offering modelled with the journeys. In this paper we will provide an account of how SAP Learning Journeys are managed and rendered, and what their evolving role is in the world of user enablement.
\end{abstract}

\section{A double bridge}

A Learning Journey is a defined path through a set of learning assets associated to a specific product, and often tailored to a specific role, structured into learning scenarios ranging from getting a first overview to becoming proficient, to extending one's skills.

Learning Journeys are visualized as interactive graphics, allowing to explore the details of the individual learning assets (each represented by a tile in the journey), even to navigate directly to the assets and begin learning (provided one already has the required access).

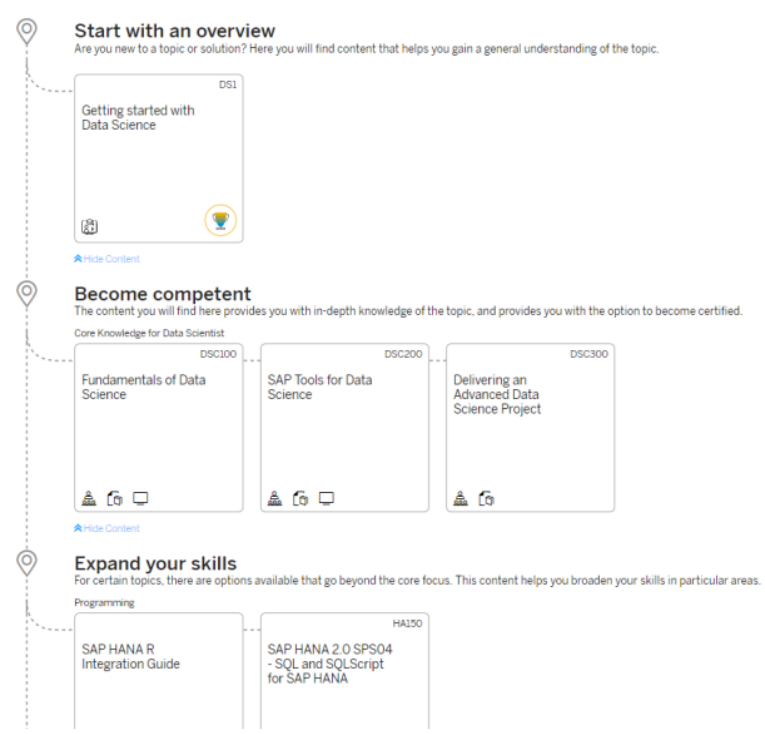

Fig. 1. Example of an SAP Learning Journey structure.

Filtering the Learning Journeys according to product, searching across all Learning Journeys for specific learning content, and within a single journey to filter by asset type are just some of the features provided with SAP Learning Journeys.

See Fig. 1 for an example of a Learning Journey structure or explore SAP Learning Journeys online on the SAP Help Portal [1].
Storing and managing the SAP Learning Journeys in a DITA content management system allows to take advantage of versioning and reuse concepts normally applied to technical documentation content, as well as automated production into different output formats, with rendering specifically adjusted to the respective channel. The Learning Journeys are rendered dynamically into SVG graphics, providing interactive features such as progressive disclosure or filtering.

On the other hand, certain technical content, for example "What's New" information for software products, has so far been included into Learning Journeys as an additional learning asset, often produced with some duplication based on the corresponding technical documentation. Including the What's New deliverables directly into the Learning Journeys eliminates the need for duplicate efforts and ties the two worlds closer together for the learner.

In this sense we have two connections between the worlds of technical documentation and learning - one on the data management side, and one on the content side. This is what we call the "double bridge" between the two worlds.

This article is not so much about ongoing or concluded research as it is an account of how this topic has evolved over the years at SAP. The author is therefore also not the only person responsible for the concepts and results, but there many other people involved in both the technical documentation and learning departments - too many to mention.

\section{Learning Journeys in the DITA landscape}

The source content for SAP Learning Journeys, including basic header information as well as required metadata, as well as the learning asset details are all maintained in the DITA content management system used for SAP technical product documentation. Each Learning Journey and each tile within the journey is represented by a single DITA topic, each supplied with

*Corresponding author: sven.leukert@sap.com 
metadata, and grouped together into DITA maps for production and publishing purposes.

Hosting the Learning Journeys in the DITA content landscape provides a lot of benefits resulting from strong content management capabilities - from complex versioning to automated translation processes, and reuse on all levels of the content. There is also the flexibility to create different flavours of Learning Journeys for different target audiences, for internal learning purposes, and as guidance maps at SAP events.

\subsection{Editing}

As Learning Journeys and tiles are represented by DITA topics, editing them is essentially the same as for technical documentation topics - typically done in an XML editor, with support for DITA semantics. However, the visual structure of the final journey (as rendered in the output) is what authors have in mind when editing a journey, and very often they are not trained technical authors, so allowing a WYSIWYG approach is desirable.

The structural data of a Learning Journey is kept in a table, and customized style sheets are used within the XML editor to represent this table in a visual structure resembling the final output structure as much as possible. Additional functions built into the stylesheet support the authors with the assignment of metadata or setting proper cross references to standardized text elements reused across all Learning Journeys.

\subsection{Production and publishing}

As with the editing, Learning Journeys are produced and published just like the technical documentation content. Fully automated build cycles pick up and produce changed content multiple times per day, and after production the output is automatically pushed to the content delivery platform (CDP) underlying the SAP Help Portal, for final validation and publication into multiple consumption channels, including SAP Learning Hub and SAP Help Portal.

The content is produced into a mix of html, JSON and image files, which are later rendered dynamically into the actual Learning Journeys.

\subsection{Rendering}

To render the Learning Journeys from the produced html and JSON files into an interactive visual appearance, a mix of custom-developed and open source JavaScript libraries is used. The main journey graphic is rendered dynamically into a collection of SVG objects, arranged on the page and equipped with interactive elements, using the open source JavaScript library D3 [2].

\subsection{Reporting}

The structure of the topics used to represent the journeys and tiles, mostly in table format, lends itself nicely to content reporting - used for internal validation processes, reuse analysis, and consistency checking.
This is done by producing the content into html files and then turning the aggregated data tables into dynamic tables, which allow filtering, sorting, searching, and export into spreadsheet format. All this is done using the open source DataTables [3] plugin for jQuery.

And as the Learning Journeys are published via the content delivery platform also used for technical documentation, its consumption reporting capabilities also automatically apply to the Learning Journey content. Predefined reports with consumption analysis information are produced automatically on a regular basis.

\section{Reuse of content in Learning Journeys}

On the non-technical side, the Learning Journeys also help to bridge the gap between the often-isolated content areas of learning and technical documentation. Separated content creation, production and publishing processes have led to some duplication of work, and unnecessary copy and paste activities.

Certain technical documentation deliverables can now be directly included into Learning Journeys as selfstudy learning assets. For example, What's New information for SAP's software products, often used by administrators and implementation consultants for planning the next upcoming projects, is now integrated into the "stay current" learning scenario of Learning Journeys as a learning asset. Likewise, blogs or videos related to specific product features and published in other channels can now be included into the product's learning journeys.

\section{References}

1. SAP Help Portal, public website, https://help.sap.com

2. Data-Driven Documents or D3.js. 4.4.0. 2016. Mike Bostock, https://d3js.org

3. DataTables. 1.10.13. 2016. SpryMedia Ltd, https://datatables.net 